\title{
THE SAFETY/SECURITY/COMMUNICATION WIRELESS LAN OF THE UNDERGROUND GRAN SASSO MOUNTAIN NATIONAL LABORATORIES OF THE ITALIAN INSTITUTE OF NUCLEAR PHYSICS
}

\author{
F. GARZIA ${ }^{1,2} \&$ R. CUSANI ${ }^{2}$ \\ ${ }^{1}$ Department of Information, Electronics and Telecommunication Engineering, SAPIENZA University of Rome, \\ Via Eudossiana 18, 000184 Rome, Italy. \\ ${ }^{2}$ Wessex Institute of Technology, Ashurst Lodge, Ashurst, Southampton, UK.
}

\begin{abstract}
Wireless networks are very useful in mobile services. In particular environments, such as the underground Gran Sasso mountain National Laboratories of the Italian Institute of Nuclear Physics they represent a vital resource for safety/security/communication. The purpose of this paper is to illustrate the design procedure, based on genetic algorithm, which has been used to design the mentioned wireless network, guaranteeing a high level of performances and reliability.

Keywords: genetic algorithm, Wireless network design.
\end{abstract}

\section{INTRODUCTION}

Wireless LANs, thanks to their capability of reaching data transmission velocities comparable with wired LANs, without the restrictions imposed by the cable itself, are actually receiving a considerable development which tends to be more rapid with time. The technologies that use wireless LAN are also named Wi-Fi, an acronym that stands for wireless fidelity.

Due to the possibilities of transmitting data with a high velocity between mobile or fixed devices without using cables, this technology allows for realizing extremely advanced services and functionalities that guarantee a high level of security [1].

Wireless LANs use electromagnetic waves at the microwave frequencies $(2.5 \mathrm{GHz}$ or $5 \mathrm{GHz}$ ), to allow point to point connections or multi-points connections. In both cases one of the devices can be represented by an access point (AP) that allows the wireless devices to connect to the wired LAN where the AP is connected.

It is evident that the design and the realization of a wireless LAN that must guarantee an optimal performance from the velocity and the benefits/costs ratio point of view (avoiding installing of an excessive number of APs that would increase the costs and decrease the reliability of the LAN due to excessive number of installed devices) needs a preliminary analysis from the service and the electromagnetic environmental point of view. In fact this last term can represent a potential source of electromagnetic noise and provoke performance decrements of the LAN.

This activity must be executed with an extreme care while dealing with environments which impose severe restrictions, such the one considered in this study.

In the considered environment, the wireless LAN must be characterized by two peculiar characteristics, represented by positioning and reliability.

In fact, since the wireless LAN must ensure positioning capabilities of the wireless terminals, each part of the underground laboratories must be covered by almost three different APs, ensuring positioning functionalities.

Further, since the wireless LAN must be characterized by a high reliability, two distinct wired LANs that serve APs have been designed. In case of malfunctioning of one wired LAN 
the other LAN continues to ensure its continued services to the APs connected to it. In this way, even if part of the positioning functionalities are lost, since it is not guaranteed that is each point of the laboratories is covered by three different APs, the wireless LAN is still capable of ensuring its basic safety/security/communication services and therefore a certain level of reliability.

The purpose of this paper is to illustrate the optimal design procedure that has been used in the considered underground Gran Sasso mountain National Laboratories using advanced techniques such as genetic algorithms (GAs) [2-7].

The optimized design procedure represents only a basic design procedure that does not consider the physical layer characteristics such as radio wave propagations, interferences, shadowing and channel selections of wireless LAN system. It generates a first approximation solution that can be refined, in a second time, considering also the physical layer characteristics.

\section{THE GRAN SASSO MOUNTAIN}

The Gran Sasso ("big rock") mountain is located in the center of Italy, about $120 \mathrm{~km}$ east of Rome, between the L'Aquila city province and Teramo city province, in the Abruzzo region.

It belongs to a system of mountains that practically separates the Adriatic sea (east) from the Tirrenian sea (west) in the middle of Italy.

The higher peak of Gran Sasso mountain is represented by Corno Grande ("big horn"), which is about $3000 \mathrm{~m}$ above sea level.

Due to the elevate altitude, even if the mountain is not so far from the Adriatic sea, a permanent glacier is also present.

On the high plane of Gran Sasso mountain, named Campo Imperatore ("Emperor high plane") a sky resort is also present.

Under the Gran Sasso mountain there is a separate double highway tunnel (one tunnel for traffic in the L'Aquila - Teramo direction and one tunnel in the Teramo - L'Aquila direction). These tunnels take the traffic from the west to the east side of central Italy and vice versa and they represent a vital connection for road traffic. The length of tunnel is about $10 \mathrm{kms}$, which represents the second road tunnel of Italy, in terms of length, after the Monte Bianco ("white mountain"), which is one of the longer road tunnels internationally (even if single tunnel while the Gran Sasso is double tunnel).

A partial view of the Gran Sasso mountain from L'Aquila side is shown in Fig. 1.

Inside the mountain, the underground Gran Sasso National Laboratories (GSNL) of Italian Institute of Nuclear Physics (INFN) are also present. They are located $1400 \mathrm{~m}$ under the central rocky mass, named Eagle Mountain.

The offices and the directional center are located $1 \mathrm{~km}$ away from the Assergi highway exit (in the L'Aquila city province) and they extend on a $12,000 \mathrm{~m}^{2}$ surface while some technical installations (fanning, electrical supply, etc.) are located on the other side of Gran Sasso mountain (in the Teramo city province) just outside the highway tunnel, at a site named Casale S. Nicola.

The entrance of GSNL is located in the Teramo - L'Aquila direction tunnel using a passage reserved to the laboratory traffic, created by means of narrowing of about $1 \mathrm{~km}$ of the tunnel road that corresponds to the underground laboratories.

The GSNL are the biggest and most important underground laboratories of the world characterized by a unique environment for the kind of research that is done inside. Further, they have been specially created for that purpose and not by renovating or adapting to already existing structures, such as active or closed mine (KAMIOKANDE in Japan and SNO in Canada). 


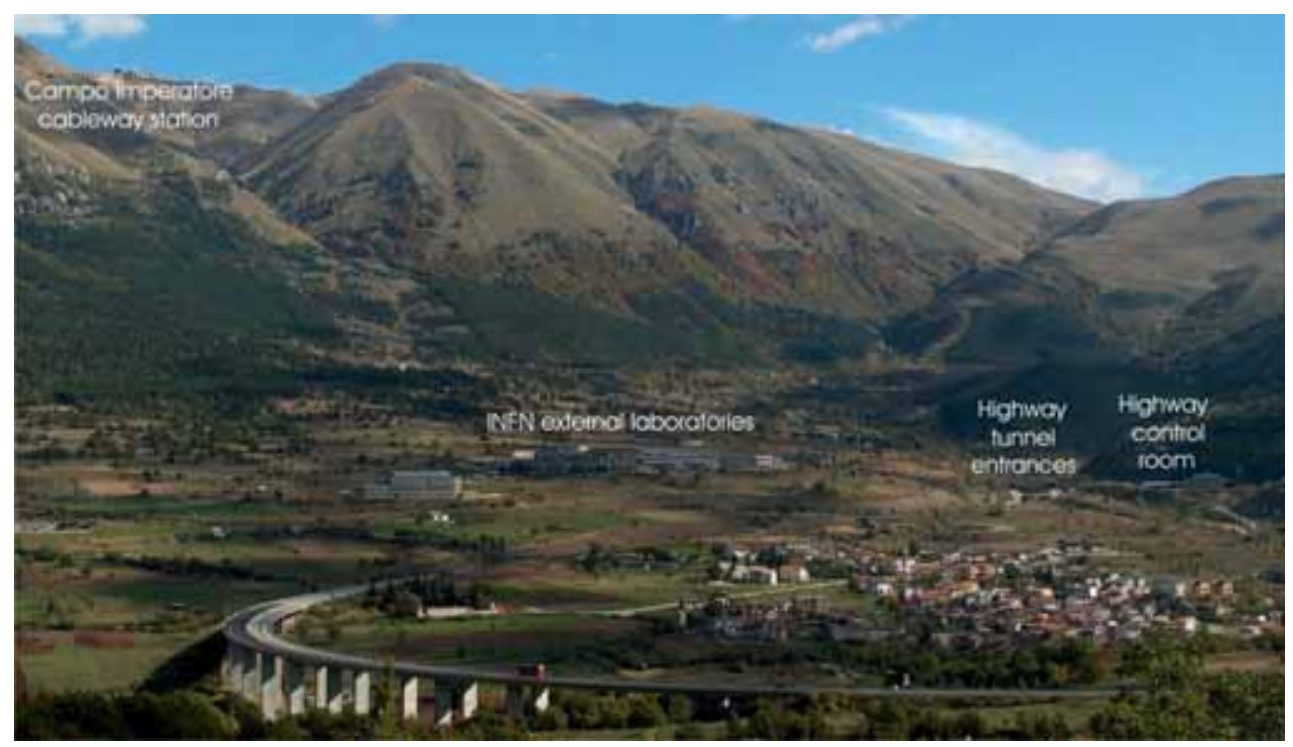

Figure 1: Partial view of Gran Sasso mountain from L'Aquila side.

The design, the approval and the public financing have been possible thanks to the simultaneous drilling and construction, in '70-' 80 years, of the highway tunnels in the same zone. The GSNL realization started in 1982 and the construction of the first experimental apparatus started only 4 years later, in 1986, when the first tunnel was opened to the public traffic. The underground laboratories are mainly constituted by three experimental rooms, whose dimensions are about $100 \times 20 \times 20 \mathrm{~m}$, and by a series of connection tunnels that are used for the installations necessary for the correct functioning of the laboratories and for hosting secondary and reduced dimension experimental devices. The total internal volume is about $180,000 \mathrm{~m}^{3}$. A 3D view of laboratories is shown in Fig. 2 and Fig. 3.

GSNL represent the ideal environment for experiments concerning neutrinos since the large amount of rock above them shields the most of nuclear particles coming from the sun and from the cosmos and let only pass neutrinos that tend to interact very rarely with matter.

Actually there are 19 experiments working in the three experimental rooms and in some connection tunnels whose name are: Borexino, COBRA, Cresst; Cuore, Dama, Libra, ERMES, GERDA, Gigs, ICARUS, Luna, LVD, OPERA, Pulex2, Tellus, Underseis, VIP, WARP R\&D, XENON.

The research in the GSNL is strictly connected to the CERN (French acronym of Centre Européen pour la Recherche Nucléaire that means European Centre for Nuclear Research) of Geneve in Switzerland where there is a neutrinos generator that shoots a neutrinos beam toward GSNL, traveling $730 \mathrm{~km}$ under the ground.

Recently OPERA experiment seems to indicate that the neutrinos coming from CERN travel at a velocity 20 parts per million above the speed of light, nature's cosmic speed limit. Given the potential far-reaching consequences of such a result, independent measurements are needed before the effect can either be refuted or firmly established. This is why the OPERA collaboration has decided to open the result to broader scrutiny.

It is therefore evident that in the Gran Sasso mountain are present a lot of subjects that make specific activities (INFN laboratories, highway, aqueduct, etc.) and for this reason the 


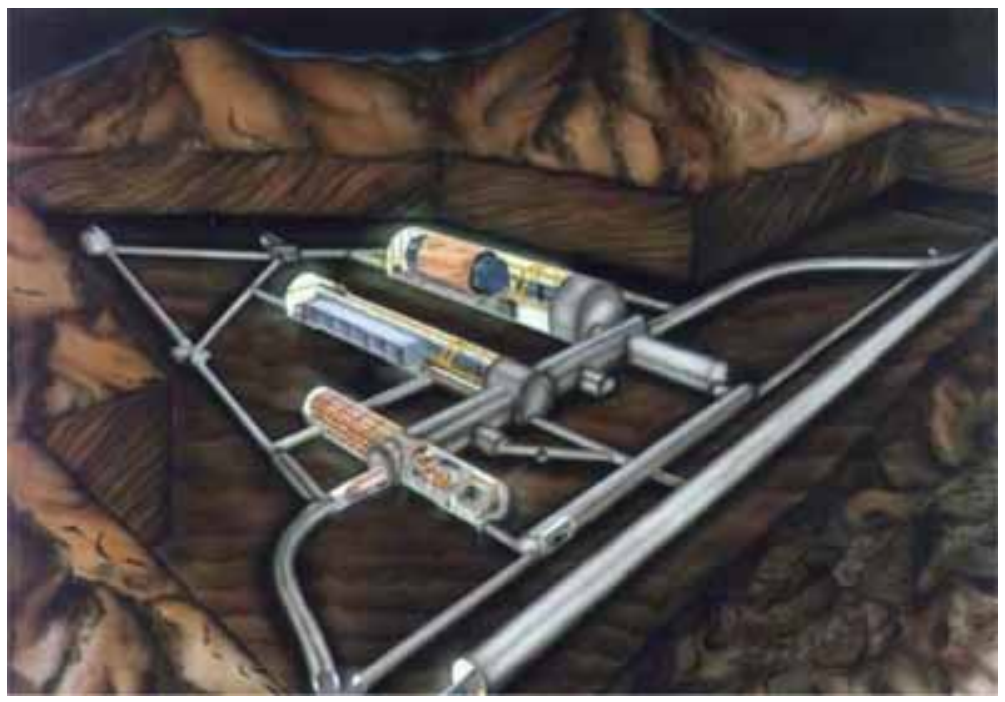

Figure 2: 3D view of the laboratories and part of highway tunnels.

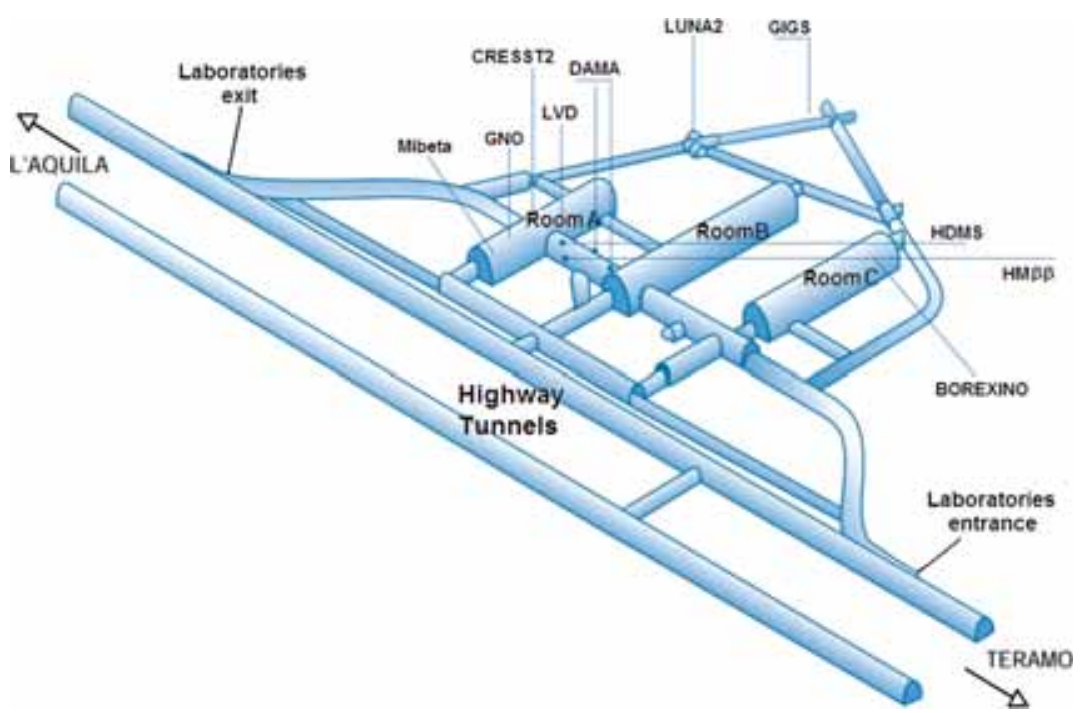

Figure 3: 3D view of the laboratories with part of the existing experiments.

Gran Sasso mountain represents a wide and complex system where each component interacts, unavoidably, with the other components. In fact, for example, the highway represents the only entrance to the laboratories, where not only people but also all the installations (such as electrical, fanning, cooling and telecommunications) that guarantee the correct functioning and the safety of laboratories, must pass through. This implies that a possible accident inside the highway tunnels can compromise not only the stability and reliability of the installations of laboratories but also the capability for fire brigades, highway tunnel personnel and 


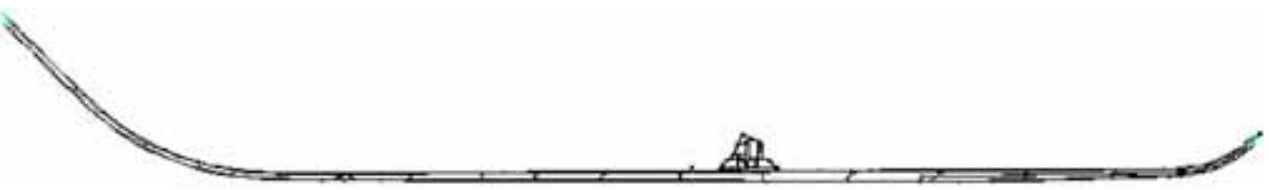

Figure 4: Plant of highway tunnels with INFN laboratories (in the middle, upper side). L'Aquila city direction is on the left side while Teramo direction is on the right side. Tunnels are about $10 \mathrm{kms}$ long.

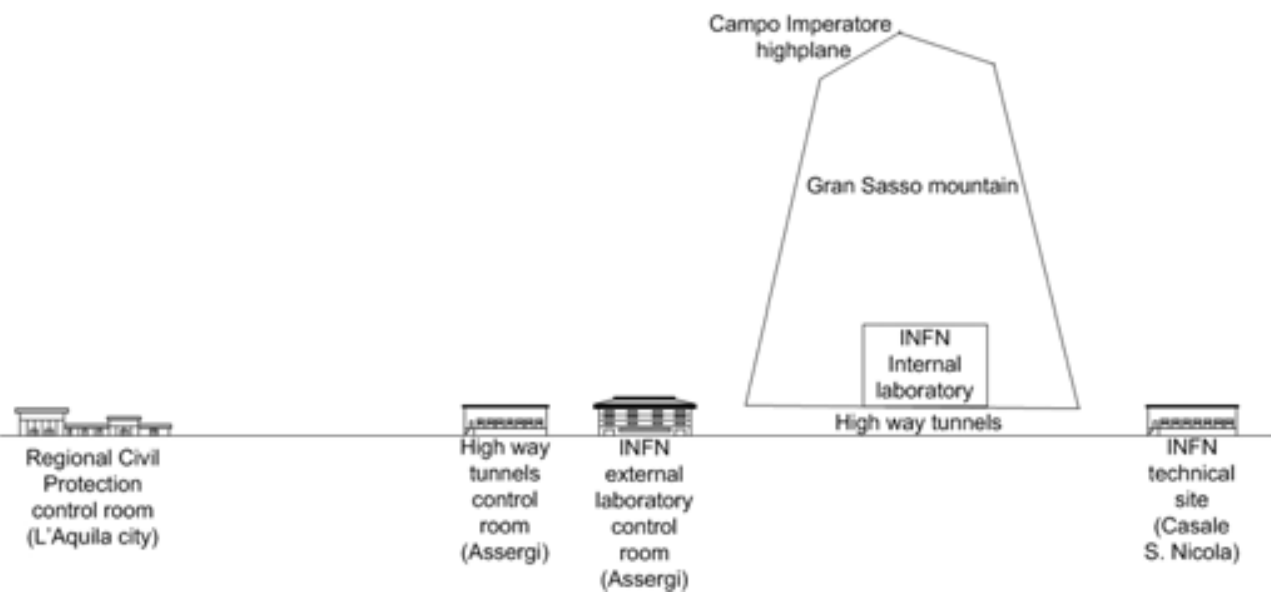

Figure 5: Transversal schematization of Gran Sasso mountain system.

emergency teams reaching the laboratories. The same happens if an accident takes place inside the laboratories. For this reason the emergency plans related to one of the subjects which operates inside the Gran Sasso mountain must also consider the other present subjects.

This characteristic gives Gran Sasso mountain an extreme oneness at the international level.

The plant of highway tunnels with INFN laboratories is shown in Fig. 4.

Due to the multitude of people, systems, devices and installations that must be controlled which must communicate with each other, it is evident that the Gran Sasso mountain, to be managed securely in the best way, needs to use intensively advanced technologies finalized to obtain a high and efficient quality of functionality, performances and services [8].

To increase the safety and security level of people that operate in Gran Sasso and to protect the unique natural environment, in July 2003 the Italian Government, through the Department of Civil Protection, designated a Delegate Commissioner of Government.

The safety/security wireless LAN presented in this paper was studied and designed by the authors, as part of a wider safety/security/communication system [8], during their activity of consultant of Delegate Commissioner of Government, concerning the safety and security aspects of Gran Sasso. The transversal schematization of the Gran Sasso mountain is shown in Fig. 5.

Due to the complexity of Gran Sasso mountain and of the subjects that operate in it, the safety/security/communication system [8] represents a unique object at the international level in terms of functionalities and reliability, greatly increasing the safety and security of people and the surrounding environment.

The scope of the paper is to illustrate the safety/security/communication wireless LAN, the GAs-based technique used to design it in an optimal way and the results obtained. 
The safety/security/communication system of the Gran Sasso mountain system is aimed at improving safety, security, communication and emergency management in a totally automatic and immediate way or at aiding the personnel by means of an expertise information system, allowing the control and activation of any component present in Gran Sasso and connected to the integrated system, by means of local or remote consoles.

The system is not only aimed at integrally control safety, security, communication and emergency but also at providing a series of advanced services (such as an innovative radio communication system) to the whole community that operates inside it or on it or lives in the surroundings.

The considered integrated system allows the maximum integration and communication of all the installations, devices and systems present in the Gran Sasso, both inside or outside it, and it guarantees their control and management in a totally automatic way, according to predefined procedures.

The system includes an advanced radio communication and localization system, capable of operating both inside and outside Gran Sasso mountain, covering a wide area that extends from L'Aquila city to Teramo city and passing above it through Campo Imperatore high plane. This radio communication system, extremely innovative, is capable of ensuring an instantaneous link between all the subjects involved in safety, security and emergency situations and can be used, in ordinary conditions, from all the enabled subjects that operates inside, outside or in the nearby of Gran Sasso mountain.

The system integrates the following components present in Gran Sasso mountain:

1. GSNL laboratories:

a) radio communication and localization of safety, security and emergency personnel;

b) wireless communication, localization and advanced information service of personnel;

c) video surveillance TV (internal and external);

d) access control;

e) anti-intrusion;

f) public address;

g) video information service;

h) internal parking management system:

i) interface with incidental lost liquid monitoring system, water source quality monitoring, fanning system, cooling system, experimental devices operating in the laboratories, electrical supply installations, fire and dangerous gas monitoring systems, environmental monitoring systems;

2. highway tunnels:
a) radio communication and localization of safety, security and emergency personnel;
b) predisposition for cellular phone communication system installation;
c) video surveillance TV;
d) optical fiber fire sensor;
e) interface with technical installations, traffic management system, environmental monitoring systems;

3. external:
a) radio communication and localization of safety, security and emergency personnel;
b) interface with various systems and installations, environmental monitoring systems.

The system guarantees a high degree of integration between the GSNL subsystem, the highway tunnels subsystem and the external subsystem, ensuring a correct and immediate 
control of all data and significant events for safety, security and emergency of Gran Sasso mountain.

In this way the system has been designed in a manner whose functionalities are really superior with respect to the functionalities of single subsystems, devices, installations or elements.

The system operates thanks to an advanced telecommunication subsystem, characterized by high reliability that is capable of working in the presence of the severe climate conditions present inside and outside the mountain.

The designed system is characterized by a high degree of modularity and expandability so that it is possible, in future, to add and integrate any other installation, device or apparatus in any point, inside or outside the Gran Sasso mountain, guaranteeing always the full control of any component present in GSNL, highway tunnels or any other place.

The integrated system concentrates the alarm signaling generated by the various installations and devices in four control rooms, unifying the management procedures and optimizing the needs of personnel resources necessary for organization and maintenance.

The four control rooms are:

1. internal GSNL;

2. external GSNL;

3. external high way tunnels;

4. regional Civil Protection (L'Aquila city).

The system is extremely modular and flexible and allows us to add, at any time, other control rooms or management consoles, according to specific needs that could appear in future.

The control room of regional Civil Protection is designed to transmit, in normal or emergency situations, data or alarm signaling to other subjects such as:
1. National Civil Protection;
2. fire brigades;
3. police;
4. aqueducts management societies;
5. national park guard;
6. regional water authority;
7. Abruzzo regional administration;
8. L'Aquila provincial administration;
9. Teramo provincial administration;
10. L'Aquila prefecture
11. Teramo prefecture;
12. local town administrations;

and any other subjects that could be individuated in a second time and located even at great distance from Gran Sasso mountain.

The functional block diagram of the integrated system is shown in Fig. 6.

The correct integration of installations and devices is reached coordinating the design of installations with the operative needs of laboratories and highway tunnels, from one side, and improving the use of each technological components to better use their functional features, from the other side. 


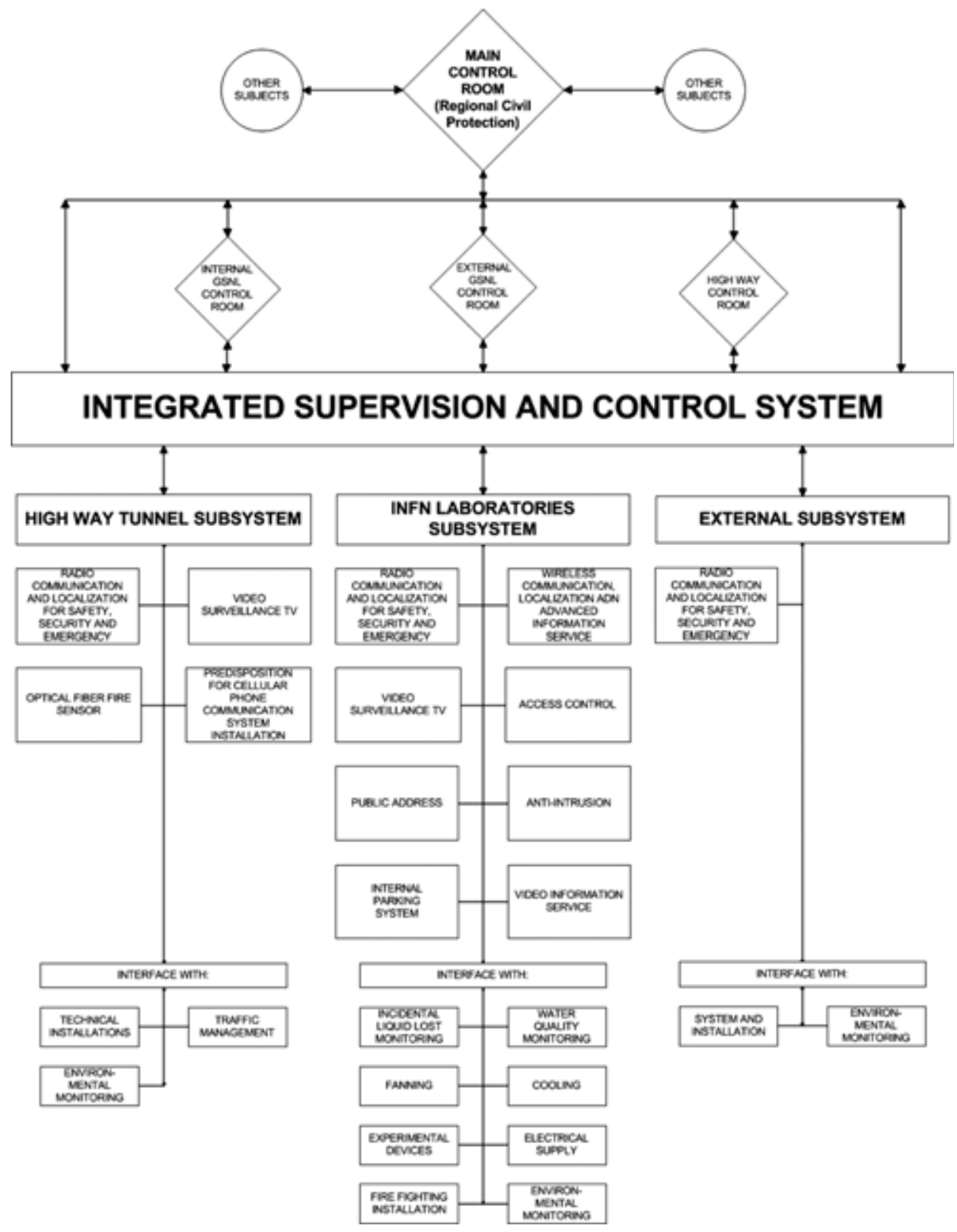

Figure 6: Functional blocks diagram of the integrated system.

The video surveillance is therefore used, for example, to view the zone where there is an alarm signaling, restricting the physical movement of safety and security personnel only to the real needs, increasing their efficiency and at the same time their safety, keeping them far away from dangerous situations.

The supervision system works according to proper manuals of procedures agreed with Civil Protection, interested Prefectures, Laboratories, highway society and all the other interested subjects. 
The consoles located in the different control rooms can manage, according to the needs and the assigned operative level, every component of the system, acting only as display units or as operative activation units. These consoles can be located even at a great distance from Gran Sasso, thanks to the transmitting capabilities owned by the telecommunication subsystem.

The system also allows the enabled radio units (radio communications or wireless) to operate as mobile consoles.

\section{THE WIRELESS SAFETY/SECURITY/COMMUNICATION WIRELESS LAN OF THE UNDERGROUND LABORATORIES}

The safety/security wireless LAN, to be installed inside GSNL, is aimed at ensuring the communication between any devices, fixed or mobile, without using cables that would unavoidably increase the complexity of the system and reduce its reliability.

The wireless LAN allows the realization of new and efficient functionalities, mainly in terms of safety and security of people and surrounding environment.

This wireless system uses light portable terminals carried by any person who goes in the underground laboratories. This system uses the wireless LAN, distributed all over the internal and external laboratories, which allows localization of all the people and any kind of communication (voice, external and internal phone, video, data, etc.). The wireless system allows the implementation of advanced services, including the capability of controlling, if the user is enabled, specific components of the integrated system, else executes an alarm.

To increase the safety level of people inside the laboratory, in case of malfunctioning of a wireless terminal, the use of reduced weight wireless identification device (WID) that sends a signal to the wireless LAN periodically is also planned. The wireless system is therefore able to localize the owner of WID in a totally autonomous way with respect to the wireless terminal. In this way it is always possible to localize, with high precision, all people inside the laboratories in case of emergency evacuation. The wireless network is quite critical and, to reach its advanced performance, it has been purposely designed using genetic algorithms [2-7].

The wireless LAN is characterized by two peculiar characteristics, i.e. positioning and reliability, as already mentioned in the introduction.

\section{DEFINITION OF THE PROBLEM}

The use of genetic algorithms for optimal placement of telecommunication resources has been studied in different context [9-30] and have demonstrated to be very useful in a situation discussed above.

In our study we deal with peculiar restrictions such as the possibility of installing the APs only on the lateral sides of the galleries of GSNL and not in any other position, and the unavailability of some installation zones due to the presence of other devices or installations. This implies that the coverage diagram of AP restricts from circular shape to half circular shape.

Further a full redundancy of the whole coverage area, that is each point of the laboratory must be covered by almost three APs, is necessary since positioning functionalities must be ensured. Since the wireless LAN must be characterized by high reliability, each zone of the laboratory needs to have two LANs located on opposite sides and the APs alternatively connected to them, so that a malfunctioning of one LAN, and of the related wireless APs, is immediately recovered by the other LAN and the related wireless APs connected to it. 


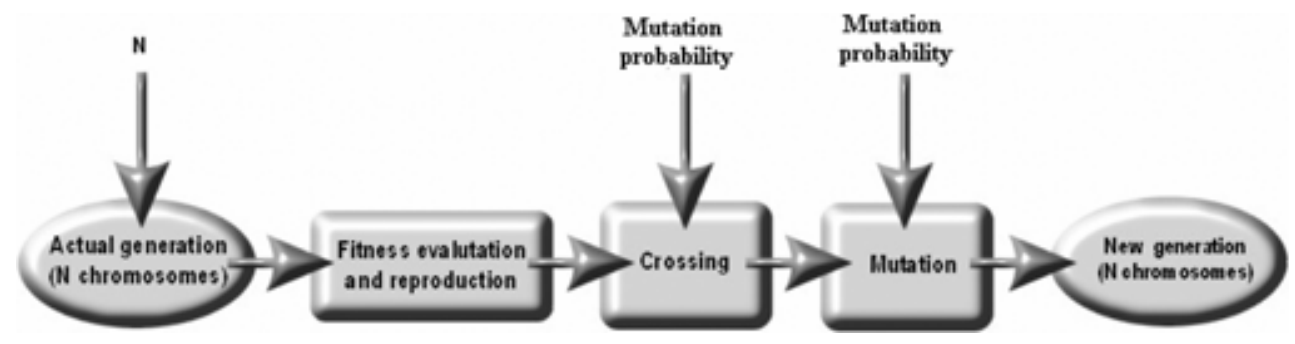

Figure 7: Flow chart of GAs operative process.

Further it is necessary to consider the background noise that could be present inside the laboratories which could reduce the coverage area of each AP and the related transmission velocity.

In the following we describe how this peculiar problem is coded and solved in terms of GAs, which allow us to solve the problem rapidly and efficiently.

\section{GENETIC ALGORITHMS}

Genetic algorithms are considered as wide range numerical optimization methods that use the natural processes of evolution and genetic recombination [2-7]. Thanks to their versatility, they can be used in different fields and find a lot of applications in wireless network optimization problems [11-30].

GAs are particularly useful when the goal is to find an approximate global minimum in a high-dimension, multi-modal function domain, in a near-optimal manner. Unlike the most optimization methods, they can easily handle discontinuous and non-differentiable functions.

The algorithms encode each parameters of the problem to be optimized into a proper sequence (where the alphabet used is generally binary) called a gene and combine the different genes to constitute a chromosome. A proper set of chromosomes, called population, undergoes the Darwinian processes of natural selection, mating and mutation, creating new generations, until it reaches the final optimal solution under the selective pressure of the desired fitness function.

GA optimizers, therefore, operates according to the following nine points:

1. encoding the solution parameters as genes;

2. creation of chromosomes as strings of genes;

3. initialization of a starting population;

4. evaluation and assignment of fitness values to the individuals of the population;

5. reproduction by means of fitness-weighted selection of individuals belonging to the population;

6. recombination to produce recombined members;

7. mutation on the recombined members to produce the members of the next generation;

8. evaluation and assignment of fitness values to the individuals of the next generation;

9. convergence check.

The flow chart of GAs operative process is schematized in Fig. 7.

\section{IMPLEMENTATION OF THE PROBLEM}

The 2D map of GSNL is shown in Fig. 8. In the same map the zones where some obstacles are present that do not allow the installation of APs are also shown. 


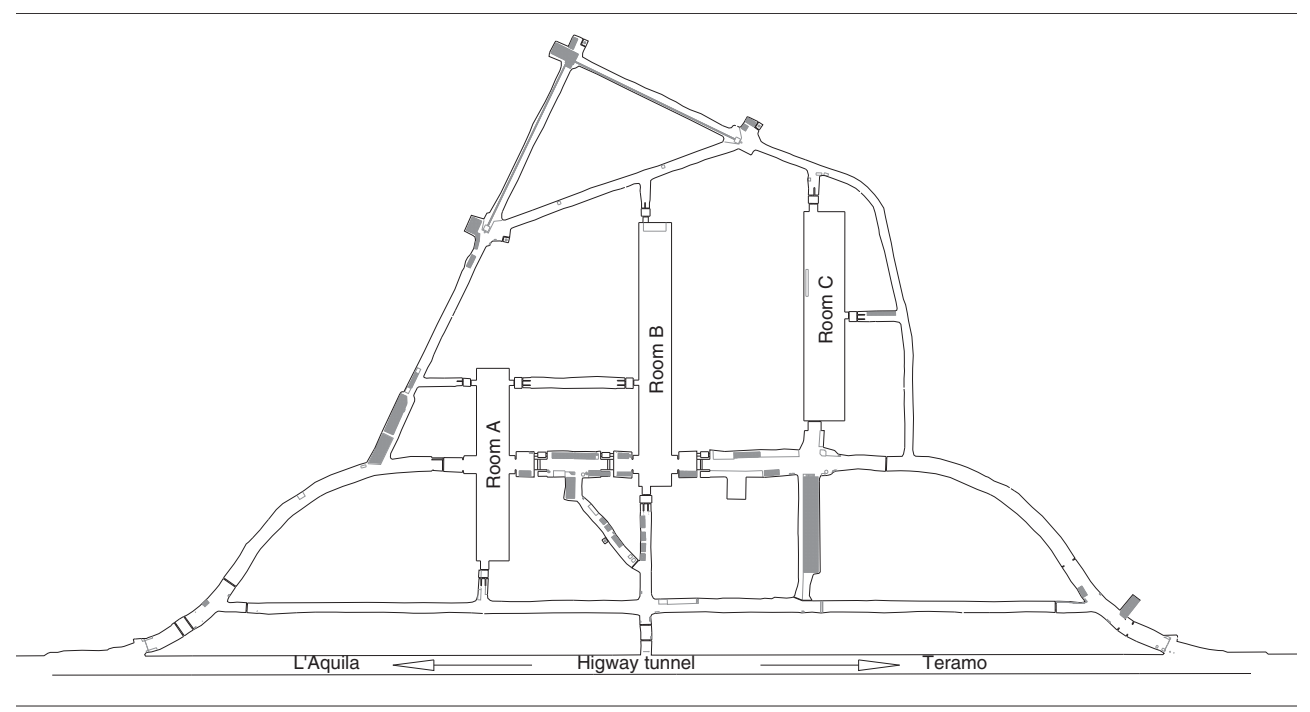

Figure 8: 2D map of Gran Sasso mountain National Laboratories.

The analysis and measurement of the background noise that could be present inside the laboratories which reduces the coverage area of each AP and the related transmission velocity was made and it did not reveal particular critical points to be considered inside the design procedure.

Once the area to be covered whose value is $A_{T}$ is calculated, and the maximum coverage distance $\mathrm{R}_{\mathrm{AP}}$ of an $\mathrm{AP}$ (which is the circular diagram that indicates where the emitted signal is above a minimum receivable threshold which is obviously related to the irradiation diagram and the emitted power) whose area is $\mathrm{A}_{\mathrm{AP}}=\pi \mathrm{R}_{\mathrm{AP}}^{2}$ is given, the minimum number $\mathrm{N}_{\text {min }}^{\mathrm{AP}}$ of access points is equal to:

$$
\mathrm{N}_{\text {min }}^{\mathrm{AP}}=(2 \cdot 3) \cdot \text { round }\left(\frac{\mathrm{A}_{\mathrm{GSNL}}}{\pi \mathrm{R}_{\mathrm{AP}}^{2}}\right)
$$

where the rounding operation is made toward the nearest integer equal or greater than the argument of the operation.

Number 2 is present due to the installation of APs on the sides of the laboratories that reduces the coverage area since only half circle of the coverage diagram is used inside the laboratories and the other half is lost inside the rocks, due to the absence of nearby rooms.

Number 3 is present due to the fact that each point of the laboratories must be covered by almost three different APs.

The number obtained from eqn.(1) is obviously ideal since it can be really reached if all the internal area is available for APs' placement and if the coverage diagram is characterized by a regular shape (e.g. square) that allows to ensure a perfect matching between the coverage of nearby APs. It is evident that in real conditions, the minimum number of APs necessary to ensure the complete coverage of the GSNL with the desired redundancy is obviously greater than the value calculated by means of eqn. (1), due to non-perfect matching of the coverage diagram of near APs and the limitation of places for APs' installation.

For this reason, given the internal area, an initial number $n * \mathrm{~N}^{\mathrm{AP}}{ }_{\min }$, of APs (where $\mathrm{n}$ is a parameter, greater than 1 , to choose at will that in our case is equal to 2) greater than the 
minimum number $\mathrm{N}^{\mathrm{AP}}$ min is considered, leaving the duty of optimizing and reducing their number to the GA, according to the availability of installation places, reaching eventually the value of $\mathrm{N}_{\text {min }}^{\mathrm{AP}}$ in ideal conditions.

Once the initial number $\mathrm{n}^{*} \mathrm{~N}^{\mathrm{AP}}{ }_{\text {min }}$ of APs is defined, it is necessary to define the parameter to be optimized for each AP which is represented by its coordinates. To increase the optimization capability of used GA, two different options have been considered: fixed range APs and variable range APs, where it is possible to regulate the emitted power of each AP to reduce linearly the maximum radius of coverage radius $\mathrm{R}_{\mathrm{AP}}$ up to zero. In this second situation the GA is capable of performing a fine matching of coverage diagram of nearby APs.

Since not all the initial APs are used to perfectly cover the considered territory, it is necessary to add, for each AP, an information that indicates if the AP is active or not.

Since it is possible to use both fixed range APs and variable range APs, two different studies were made to consider both situations.

\subsection{Fixed range APs}

Fixed range APs are characterized by a fixed coverage area that cannot be changed by the final user.

In this situation three solution parameters, for each AP, must be considered. They are:

1. x coordinate;

2. y coordinate;

3. activity of the AP.

Let us discuss now the variability range of the parameters indicated above and the relative accuracy necessary to represent them in terms of binary strings.

Concerning the $\mathrm{x}$ and $\mathrm{y}$ coordinates, if we choose a $1 \mathrm{~m}$ resolution and we consider the maximum extension of laboratories $(\approx 1 \mathrm{~km}), 9$ bits are enough to represent a distance variable between 1 and $1.023 \mathrm{~m}$ (i.e. $\approx 1 \mathrm{~km}$ ).

The activity of each AP is coded using a single bit, where binary 1 indicates that the Base Station is active while binary 0 indicates that the BS is not active, even if it is located in a given $(\mathrm{x}, \mathrm{y})$ position.

Three genes are therefore used to encode the parameters of each AP whose total length is equal to 19 bits. The genes features are summarized in Table 1.

Each chromosome, or individual, representing a solution of the problem, is composed by a string representing all the $\mathrm{n}^{*} \mathrm{~N}^{\mathrm{AP}}{ }_{\text {min }}$ APs and the related three parameters (whose total length is equal to 19 bits). The total length of each chromosome is therefore equal to $19^{*} \mathrm{n}^{*} \mathrm{~N}^{\mathrm{AP}}{ }_{\min }$ bits.

Table 1: Features of the three genes used to identify a fixed range AP.

\begin{tabular}{clcc}
\hline \multicolumn{4}{c}{ Fixed range AP parameters } \\
\hline Gene & Feature & Number of bits & Range \\
\hline 1 & x coordinate & 9 & $0-1.023 \mathrm{~m}$ \\
2 & y coordinate & 9 & $0-1.023 \mathrm{~m}$ \\
3 & Activity of AP & 1 & $0-1$ \\
\hline
\end{tabular}


It is now necessary to define the fitness function $\mathrm{f}$. This function must consider all the desired optimization goals that are:

1. integral coverage of laboratories with the minimum number of APs;

2. overlapping of coverage diagram by means of almost three APs belonging to different fixed LANs, to increase as more as possible the reliability of the wireless LAN and ensure positioning capabilities;

3. placement of APs only in the allowed zones;

4. the APs must be connected to the two different fixed LANs.

Points one and two are synthesized with a proper function while point three is considered using a proper territorial array.

The considered fitness function of the generic chromosome $\mathrm{C}$ can be expressed as:

$$
\mathrm{f}(\mathrm{C})=\frac{\alpha \frac{\text { coverage area }(\mathrm{C})}{\text { total coverage area }}+\beta \frac{3 \text { APs overlapped area }(\mathrm{C})}{\text { total coverage area }}}{\frac{\mathrm{N}^{\mathrm{AP}}(\mathrm{C})-\mathrm{N}_{\text {min }}^{\mathrm{AP}}}{\mathrm{N}_{\text {min }}^{\mathrm{AP}}}+1}
$$

where "coverage area (C)" is the area covered by the APs distribution related to the chromosome $\mathrm{C}$, or individual $\mathrm{I}, \mathrm{N}^{\mathrm{AP}}(\mathrm{C})$ is the number of active APs related to the chromosome $\mathrm{C}$ and " 3 APs overlapped area (C)" is the total area of overlapping of three different coverage diagrams belonging to three different APs to increase the reliability of the wireless LAN and to ensure positioning capabilities (three spatial information).

The mentioned function keeps into consideration the performances of the chromosome $\mathrm{C}$ (APs distribution) in terms of coverage area (first term of numerator), three overlapping APs (second term of numerator) and reduced number of APs (denominator). The number 1 that has been added as a second term of the denominator is necessary to avoid divergence toward infinity when the fitness function is used to evaluate a chromosome $\mathrm{C}$ that uses a minimum number $\mathrm{N}_{\text {min }}^{\mathrm{AP}}$, of APs.

The parameters $\alpha$ and $\beta$ are used to control the evolution force in terms of coverage area $(\alpha)$ and coverage of each point of GSNL by the different APs $(\beta)$. Different values have been tried but the best results, in terms of optimal solution and reduction of the computation time, are obtained if $\alpha$ is variable between 1 and 2.2 and $\beta$ is variable between 1.5 and 2.8. These values ensure a good balance between the need of covering the whole area of the GSNL and the same area by almost three different APs in each point.

The information relative to the allowed zones for APs' placement is stored in a proper binary array, characterized by the same dimensions and resolution of $(\mathrm{x}, \mathrm{y})$ coordinates of APs (i.e. $2^{9} \times 2^{9} \mathrm{~m}$ ). Each element of the array (representing a cell of the laboratory whose dimensions are $1 \mathrm{~m} \times 1 \mathrm{~m}$ ) that can be used for APs' placement is marked with binary 0 while if it cannot be used for APs' placement, it is marked with binary 1. Practically, inside the mentioned array the internal profile of the laboratories is stored, with the exception of the zones occupied by devices that do not allow the installation of APs.

The control of the APs' placement in not allowed zones is made at any genetic operation (reproduction, crossing, mutation), checking in the proper array if the coordinate of the APs of the actual chromosome $\mathrm{C}$, or individual I, are marked with binary 1: if this happens, the related chromosome is deleted. 
The control about the coverage of any point of the laboratories by almost one AP belonging to a single LAN is made at any genetic operation (reproduction, crossing, mutation) for the two wired LANs: if this does not happen, the related chromosome is deleted. In this way the wireless coverage of the whole laboratories in case of malfunctioning of one of the two wired LANs is ensured, loosing positioning capabilities (due to the lack of coverage of three APs) but conserving the basic services.

The control about the coverage of any point of the laboratories by almost three different APs belonging to the two different LANs is made at any genetic operation (reproduction, crossing, mutation): if this does not happen, the related chromosome is deleted.

The control about the connection of the APs to the two different fixed LANs is made at any genetic operation (reproduction, crossing, mutation): if more than $60 \%$ of APs is connected to only one fixed LAN, the related chromosome is deleted. This ensures that $50 \%$ of the installed APs are connected to one fixed LAN while the other $50 \%$ are connected to the other fixed LAN. This quantity can vary between $-10 \%$ and $+10 \%$.

Once the initial population at random is generated, the individuals characterized by APsnot-allowed-placement are eliminated, and the selection is operated only on the remaining individuals, until attaining a reproduced population characterized by the same number of individuals of the initial population.

Since the initial population is initialized at random, there is generally a portion of it that is eliminated at the beginning, but after the first iterations more fitting individuals are generated and it is not necessary to eliminate any of them.

Once the population is recombined and mutated, the fitness function of the population is again calculated with the same criteria illustrated above, considering only fitting individuals. The converge test is made controlling if the difference between the mean value of fitness functions of the valid individuals belonging to the actual generation and the mean values of the last $\mathrm{N}_{\mathrm{G}}$ generations is lesser than a certain percentage value $\mathrm{p}_{\text {stop }}$.

Good results and rapid converge are obtained with population composed by 50-60 individuals, with converge test parameters $\mathrm{N}_{\mathrm{G}}$ and $\mathrm{p}_{\text {stop }}$ equal to 25 and 0.08 respectively.

\subsection{Variable range APs}

Variable range APs are characterized by a variable coverage area that can be regulated in the installation phase and that can be changed any time.

In this situation it is necessary to consider four solution parameters, for each variable range AP, that are:

1. $\mathrm{x}$ coordinate;

2. y coordinate;

3. activity of the AP;

4. reduction of maximum coverage distance $\mathrm{R}_{\mathrm{AP}}$

Concerning the first three parameters, the range variability is similar to the previous situation.

Concerning the last parameter, the reduction of maximum coverage distance can vary between 0 and 100\%: we choose to use 7 bits that allow us to represent 127 numbers. Since the necessary numbers to codify the percentage with a resolution is equal to $1 \%$ and are only 100 , the remaining 27 values are used to represent the corresponding percentage values between $0 \%$ and $27 \%$, maintaining them active in the evolution process. A 6 bits encoding is 
Table 2: Features of the four genes used to identify a variable range AP.

\begin{tabular}{clcl}
\hline \multicolumn{4}{c}{ Variable range AP parameters } \\
\hline Gene & Feature & Number of bits & \multicolumn{1}{c}{ Range } \\
\hline 1 & x coordinate & 9 & $0-1.023 \mathrm{~m}$ \\
2 & y coordinate & 9 & $0-1.023 \mathrm{~m}$ \\
3 & Activity of AP & 1 & $0-1$ \\
4 & Reduction of maximum & 7 & $0-100 \%$ \\
& coverage distance $\mathrm{R}_{\mathrm{AP}}$ of AP & & \\
\hline
\end{tabular}

not possible since it allows us to represent only 64 numbers with a resolution of 1 , which is not enough for our purposes.

Four genes are therefore used to encode the parameters of each variable range AP whose total length is equal to 26 bits. The features of the genes are summarized in Table 2.

Each chromosome, or individual, representing a solution of the problem, is composed by a string representing all the $\mathrm{n} * \mathrm{~N}^{\mathrm{AP}}{ }_{\text {min }}$ APs and the related four parameters (whose total length is equal to 26 bits). The total length of each chromosome is therefore equal to $26^{*} n^{*} \mathrm{~N}^{\mathrm{AP}}{ }_{\text {min }}$ bits.

The fitness function and all the other considerations are similar to the previous situation.

\section{RESULTS}

The proposed GA has demonstrated to be extremely versatile in APs' optimal placement in areas, such as GSNL, where plenty of restrictions are present. The optimal solutions are generally obtained after a limited number of generations that rarely overcomes 150 iterations.

The computation time strictly depends on the number of APs considered, since each of them adds 19/26 bits to each chromosome and therefore 19/26 bits of information to be handled by the GA as a function of the considered situation (fixed range or variable range AP). The number of APs grows with the reduction of maximum coverage $\mathrm{R}_{\mathrm{AP}}$ of APs: the longer this distance the lesser the number of APs, and therefore the time necessary to reach the final optimal solution.

Since the proposed design technique must be independent from any particular commercial devices, different optimizations were made considering variable values of maximum reachable distance $\mathrm{R}_{\mathrm{AP}}$ of APs to know for which values the maximum reduction of initial number of APs is obtained. The $n$ value to be multiplied for $\mathrm{N}^{\mathrm{AP}}{ }_{\text {min }}$ was chosen to be equal to 2 . The results for the fixed range APs are shown in Fig. 9.

It is possible to see that the maximum reduction of number of APs is obtained if devices that ensure a coverage distance between 10 and $60 \mathrm{~m}$ are used. This can be explained with the geometry of laboratories. The lower value of $10 \mathrm{~m}$ results from the width of the three large experimental rooms $(20 \mathrm{~m})$ : under this value an AP installed on a side of the room cannot reach the middle of the room, ensuring its wireless coverage, since another AP located on the opposite side cannot do the same. In this situation the problem does not have a full coverage solution and the GA algorithm is not able to find it. The higher value of $60 \mathrm{~m}$ is due to the absence of wide spaces (with the exception of the main rooms) and the presence of numerous long galleries and reduced spaces: this geometrical configuration makes a short-medium range coverage AP preferable with respect to a long coverage AP, thanks to the possibility of 


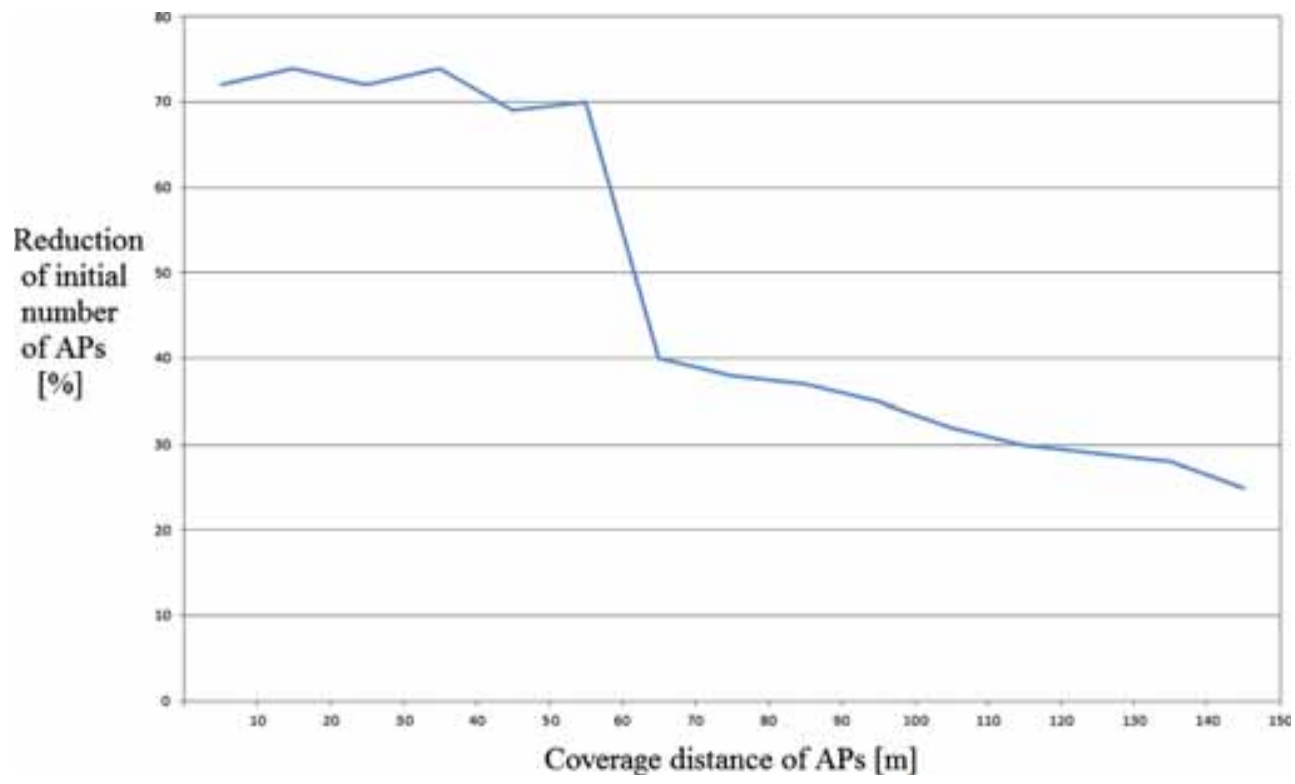

Figure 9: Reduction [\%] of the initial number of access points as a function of the maximum coverage distance $\mathrm{R}_{\mathrm{AP}}$ of access point to be installed.

GA of optimizing, in the better way, the AP installation to respect the restrictive design condition imposed. This implies that it is not necessary to choose more expensive long range APs, since a coverage between 10 and 60 m ensures optimal results in terms of reduction of number of APs and therefore in terms of increasing of reliability of the wireless LAN, ensuring, at the same time, the coverage of any point of GSNL with almost three APs, guaranteeing positioning capabilities of the wireless devices that use wireless LAN.

It is obvious that different design solutions were obtained, each of them characterized by a different placement of APs inside the laboratories and all respecting the design conditions. They are not shown for the sake of brevity.

In the variable range AP situation, the GA can select, for any AP of the solution, the optimal value, variable between $0 \%$ and $100 \%$, of the maximum range to find the optimal solution.

Different simulations with different variable maximum distances of APs were made and all the simulations gave a final optimal mean value of the coverage distance equal to about $35 \mathrm{~m}$. This represents the mean value in the range $10-60 \mathrm{~m}$ found in the previous situation, confirming the previous conclusion that, in this situation, shorter range APs are more suitable with respect to longer range APs. Further, it is not necessary to use variable range APs since the GA algorithm is capable of finding the more suitable installation positions of the given fixed range APs (that are also optimal from the reduction-of-their-number point of view if their coverage distance is variable between 10 and $60 \mathrm{~m}$ ) to reach the desired goal.

\section{CONCLUSIONS}

An efficient technique that uses genetic algorithms for APs' placement in areas with different kind of restrictions, such as Gran Sasso underground National Laboratory of Italian Institute of Nuclear Physics, has been presented. It is capable of operating in any kind of real situation, reaching optimal results. 
It can be used in the initial planning of wireless LAN, adding later further restrictions, which has been identified, to improve the found solutions.

It gives not only different optimal solutions for placement of access points inside the laboratories but also the maximum coverage requested to the APs to reach the minimum cost of installation.

The optimized design procedure represents only a basic design procedure that does not consider the physical layer characteristics such as radio wave propagations, interferences, shadowing and channel selections of wireless LAN system. It generates a first approximation solution that can be refined, in a second time, considering also the physical layer characteristics.

The use of GA techniques on this kind of problem ensures to find, always and efficiently, quasi-optimal solutions, that would otherwise be impossible to locate due to the considerable number of parameters involved in the optimization problem and due to the numerous vinculums to be considered in the resolution of the problem.

\section{REFERENCES}

[1] Garzia, F., Communication security, WIT Press (Southampton and Boston) in print, 2013

[2] Davis, L., Genetic Algorithms and Simulated Annealing, Morgan Kaufmann Publishers, Inc.: Los Altos (CA), 1987.

[3] Davis, L., Handbook of Genetic Algorithm, Van Nostrand Reinhold: New York, 1991.

[4] Dias A.H.F. \& de Vasconcelos J.A., Multiobjective Genetic Algorithms Applied to Solve Optimization Problems, IEEE Transactions on Magnetics, 38(2), pp. 1133-1136, 2002. doi: http://dx.doi.org/10.1109/20.996290

[5] Goldberg, D.E., Genetic Algorithms in Search, Optimisation and Machine Learning, Addison-Wesley: New York, 1989.

[6] Goldberg, D.E. \& Deb, K., Foundations of Genetic Algorithms, Morgan Kaufmann: New York, 1991.

[7] Holland, J.H., Genetic Algorithms, Scientific American: New York, pp. 66-72, 1992.

[8] Garzia, F. \& Cusani, R., The integrated safety/security/communication system of the Gran Sasso mountain in Italy, In Print on International Journal of Safety \& Security Engineering, WIT Press (Southampton and Boston), 2(1) pp. 13-39, 2012

[9] Garzia, F., Sammarco, E. \& Cusani, R. The integrated security system of the Vatican City State, International Journal of Safety \& Security Engineering, WIT Press (Southampton and Boston): 1(1), pp. 1-17, 2011.

[10] Contardi, G., Garzia, F. \& Cusani, R., The integrated security system of the Senate of the Italian Republic, International Journal of Safety \& Security Engineering, WIT Press (Southampton and Boston): 1(3), pp. 219-246, 2011.

[11] Cacciani, D., Garzia, F., Neri, A. \& Cusani, R., Optimal territorial resources placement for multipurpose wireless services using genetic algorithms, International Journal Wireless Engineering and Technology, 2, pp. 184-195, 2010. doi: http://dx.doi. org/10.4236/wet.2011.23026

[12] Garzia, F., Perna, C. \& Cusani, R., Optimization of UMTS network planning using genetic algorithms, International Journal Communications and Network, 2(3), pp. 193-199, 2010.

[13] Maksuriwong, K., Varavithya, V. \& Chaiyaratana, N., Wireless LAN access point placement using a multi-objective genetic algorithm, IEEE International Conference on Systems, Man and Cybernetics, pp. 1944-1949, 2003.

[14] Nagy, L. \& Farkas, L., Indoor base station location optimization using genetic algorithms, IEEE International Symposium on Personal, Indoor and Mobile Radio Communications, pp. 843-846, 2000. 
[15] Rong-Hou, W., Yang-Han, L. \& Shih-An, C., Planning system for indoor wireless network, IEEE Transactions on Consumer Electronics, pp. 73-79, 2001. doi: http://dx.doi. org/10.1109/30.920422

[16] Shih-An, C., Yang-Han, L., Yen, R.Y., Yu-Jie, Z., Chih-Hui, K., Shiann-Tsong, S. \& Meng-Hong, C., Optimal prediction tool for wireless LAN using genetic algorithm and neural network concept, APCC/OECC '99 Fifth Asia-Pacific Conference on Communications and Fourth Optoelectronics and Communications Conference, pp. 786-789, 1999.

[17] Liu, S., Zhong, C., Zhang, L. \& Zhang, L., Optimization design of wireless local area network based on improved adaptive genetic algorithm, WCICA 2006, The Sixth World Congress on Intelligent Control and Automation, 1, pp. 3357-3360, 2006.

[18] Abdelkhalek, O., Krichen, S., Guitouni, A. \& Mitrovic-Minic, S., A genetic algorithm for a multi-objective nodes placement problem in heterogeneous network infrastructure for surveillance applications, 4th Joint IFIP Wireless and Mobile Networking Conference, pp. 1-9, 2011.

[19] Sato, Y. \& Kera, Y., Wireless access point configuration by genetic algorithm using a local rule, The 2005 IEEE Congress on Evolutionary Computation, 2, pp. 1516-1523, 2005.

[20] Ming, L., Bing-kun, G., Jia, L. \& Hong, D., Research of the AP optimize method based on genetic simulated annealing algorithm, ICIC '09 Second International Conference on Information and Computing Science, 2, pp. 152-155, 2009.

[21] Hu, J. \& Goodman, E., Wireless access point configuration by genetic programming, CEC2004 Congress on Evolutionary Computation, 1, pp. 1178-1184, 2004.

[22] Xu, Y., Wang, Y. \& Ma, L., A novel WLAN indoor positioning algorithm based on positioning characteristics extraction, 2010 Fourth International Conference on Genetic and Evolutionary Computing, pp. 134-137, 2010.

[23] Yeong, S.Y., Wan, T.-C. \& Al-Salihy, W., Combination of neural network based clustering and genetic algorithm for multi-objective 802.11n planning, IEEE 9th Malaysia International Conference on Communications, pp. 852-856, 2009.

[24] Chen, S.A., Lee, Y.H., Yen, R.Y., Zheng, Y.J., Ko, C.H., Sheu, S.T. \& Chen, M.H., Optimal prediction tool for wireless LAN using genetic algorithm and neural network concept, APCC/OECC '99. Fifth Asia-Pacific Conference on Communications and Fourth Optoelectronics and Communications Conference, 1, pp. 786-789, 2009.

[25] Wu, R.H., Lee, Y.H. \& Chen, S.A., Planning system for indoor wireless network, IEEE Transactions on Consumer Electronics, 47, pp. 73-79, 2001. doi: http://dx.doi. org/10.1109/30.920422

[26] Vanhatupa, T., Hannikainen, M. \& Hamalainen, T.D., Genetic Algorithm to Optimize Node Placement and Configuration for WLAN Planning, ISWCS 2007 4th International Symposium on Wireless Communication Systems, pp. 612-616, 2007.

[27] Gordejuela-Sanchez, F. \& Zhang, J., Practical design of IEEE 802.16e networks: a mathematical model and algorithms, IEEE GLOBECOM 2008 Global Telecommunications Conference, pp. 1-5, 2008.

[28] Qing, L.X. \& Yan, T., An efficient heuristic control algorithm for wireless sensor networks, 2010 International Conference on Educational and Network Technology, pp. 545-548, 2010. doi: http://dx.doi.org/10.1109/ICENT.2010.5532097

[29] Nistal-Ariza, S., Fernandez-Duran, A. \& Alonso, J.I., Simulation based algorithm comparison for planning and optimization of indoor wireless networks, 2008 IEEE Radio and Wireless Symposium, pp. 211-214, 2008. doi: http://dx.doi.org/10.1109/ RWS.2008.4463466

[30] Qin, H. \& Du, Y., A multiobjective evolutionary tracking indoor positioning algorithm for smart space, WGEC '08 Second International Conference on Genetic and Evolutionary Computing, pp. 42-46, 2008. 